\title{
DIFICULDADES ALIMENTARES EM CRIAN- ÇAS E ADOLESCENTES COM PARALISIA CEREBRAL: REVISÃO SISTEMÁTICA
}

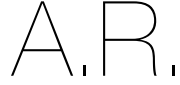
ARTIGO DE REVISÃO

${ }^{1}$ Escola Superior de Tecnologia da Saúde de Coimbra

Rua 5 de Outubro,

3046-854 Coimbra,

Portugal

${ }^{2}$ Centro de Reabilitação da Associação do Porto de Paralisia Cerebral,

Alameda de Cartes, n. ${ }^{\circ} 192$ 4300-008 Porto, Portugal

`Endereço para correspondência:

Joana Cunha

Calçada do Mineiro, n. ${ }^{3} 1$,

Sobrado,

450-154 Castelo de Paiva,

Aveiro, Portugal

joana03cunha@gmail.com

Histórico do artigo:

Recebido a 21 de julho de 2020 Aceite a 30 de setembro de 2020

\section{FEEDING DIFFICULTIES IN CHILDREN AND ADOLESCENTS WITH CEREBRAL PALSY: SYSTEMATIC REVIEW}

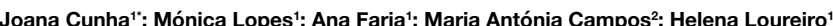

RESUMO

INTRODUÇÃO: A Paralisia Cerebral é um conjunto de distúrbios do desenvolvimento do movimento e postura resultantes de lesões cerebrais no feto ou criança. Estas alterações conduzem frequentemente a dificuldades alimentares e podem estar relacionadas com ingestão alimentar inadequada, estado nutricional, função motora, duração das refeições e dependência, qualidade de vida da criança e dos cuidadores.

OBJETIVOS: Realizar uma revisão sistemática sobre as dificuldades alimentares em crianças e adolescentes com Paralisia Cerebral e o seu impacto negativo na saúde e qualidade de vida.

METODOLOGIA: Recolher e analisar dificuldades alimentares descritas em artigos científicos, segundo as normas PRISMA. De seguida, analisar o seu impacto na saúde e qualidade de vida da população em estudo.

RESULTADOS: Um total de 19 estudos foi selecionado para esta revisão, sendo que todos relataram dificuldades alimentares. Onze referiram dificuldades alimentares de caráter oromotor, 6 identificaram problemas gastrointestinais, 8 abordaram a necessidade de assistência durante as refeições e 1 a recusa alimentar. A associação entre as competências alimentares e a função motora fol relatada em 6 estudos. Dez artigos evidenciaram um comprometimento da ingestão alimentar e 4 afirmaram que este pode conduzir à necessidade de utilização de outras vias de alimentação, como a gastrostomia. A baixa qualidade de vida destas crianças e dos seus cuidadores foi descrita em 3 estudos.

CONCLUSÕES: As dificuldades alimentares são recorrentes na população com Paralisia Cerebral. Apesar da procura de um nutricionista ser pouco frequente, este deve ser consultado assim que surjam sinais de um possível comprometimento alimentar para minimizar as suas consequências, melhorando assim o estado nutricional e a qualidade de vida.

\section{PALAVRAS-CHAVE}

Alimentação, Intervenção dietética, Nutrição, Paralisia Cerebral, Problemas alimentares

\section{ABSTRACT}

INTRODUCTION: Cerebral Palsy is a group of disorders in movement and posture development resulting from fetus' or child's brain damage. These changes often lead to feeding difficulties and may be related to inadequate food intake, nutritional status, motor function, meals length and dependence, quality of life of child and caregivers.

OBJECTIVES: To review feeding difficulties in children and young people with Cerebral Palsy and its impact on health and life quality. METHODOLOGY: Synthesize and analyze feeding difficulties described in scientific articles, based on PRISMA methods. Then, analyze the negative effect of these difficulties on health and quality of life in the pediatric population with Cerebral Palsy.

RESULTS: A total of 19 studies were selected for this review. All articles reported eating difficulties. Eleven scientific articles mentioned oromotor eating difficulties, 6 identified gastrointestinal problems, 8 described the need of assistance during mealtimes and 1 food refusal. The association between eating skills and motor function was reported in 6 studies. Ten articles revealed food intake was compromised and 4 stated that it may lead to the use of other feeding routes, such as gastrostomy. The low quality of life of these children and their caregivers was described in 3 studies.

CONCLUSIONS: Feeding difficulties are frequent in Cerebral Palsy population. Although the recommendation for consulting a nutritionist is uncommon it should occur as soon as complications arise to minimize the consequences and improving nutritional status and life quality.

KEYWORDS

Feeding, Dietary intervention, Nutrition, Cerebral Palsy, Feeding problems 


\section{INTRODUÇÃO}

A Paralisia Cerebral (PC) define-se por "um grupo de distúrbios permanentes do desenvolvimento do movimento e postura, que provocam limitações na atividade e que são atribuídas a distúrbios não progressivos que ocorrem durante o desenvolvimento cerebral do feto ou criança. Os distúrbios motores da PC são frequentemente acompanhados por distúrbios da sensação, perceção, cognição, comunicação e comportamento, epilepsia e problemas músculoesqueléticos secundários" (1). O diagnóstico é amplo e habitualmente estabelecido aos 4-5 anos de idade (2-5). A sua origem é provavelmente multifatorial, considerando-se 3 grupos de fatores: pré-natais, como infeções congénitas, gravidez múltipla ou falta de oxigenação; perinatais, como anoxia neonatal e pré-eclâmpsia; e pós-natais como infeções ou traumas (6-8).

A classificação da PC baseia-se em diferentes critérios. De acordo com a distribuição topográfica, podemos estar perante um caso de tetraplegia, diplegia, hemiplegia ou situações raras de triplegia e monoplegia (9). Quanto à descoordenação motora, a PC pode classificar-se em distónica, atáxica ou espástica, sendo este o tipo motor mais predominante $(2,9,10)$. Existem ainda as classificações mistas, já que os casos puros são raros (9). As diferentes classificações estão na origem da complexidade e diversidade desta condição (11). PC é a principal causa de incapacidade física infantil $(11,12)$. Dados mundiais do Centers for Disease Control and Prevention relatam uma prevalência que varia de 1,5 a mais de 4 por 1.000 nados vivos. Sabe-se ainda que a incidência de PC é superior no sexo masculino $(2,8,13)$. Em Portugal, segundo o último relatório do Programa de Vigilância Nacional da Paralisia Cerebral aos 5 Anos de Idade, a taxa de incidência é de 1,55 (13).

Quando comparados com a população em geral para a mesma idade e sexo, crianças ou adolescentes com PC leve parecem ter uma esperança de vida semelhante ou ligeiramente inferior. Contudo, o agravar do tipo de PC, o comprometimento motor e as competências alimentares têm impacto na esperança média de vida (14). Em relação à mortalidade, esta é cerca de 50 vezes maior que na população pediátrica geral. Este valor varia com o número e a gravidade das deficiências associadas e com fatores como sexo e idade (15-17). As maiores taxas de mortalidade correspondem a problemas respiratórios, seguidos de doenças do sistema circulatório $(15,18-20)$.

Nesta população é frequente o descontrolo motor, que resulta em movimentos involuntários e descoordenados, espasticidade muscular e disfunções oromotoras $(12,21)$. Para além destes, ocorrem lesões no sistema nervoso central que podem conduzir a epilepsia e convulsões, distúrbios da sensação, perceção, cognição, comunicação e comportamento, assim como dor, défices auditivos e visuais. Em alguns casos, podem surgir problemas ósseos, articulares, urinários, pulmonares, endócrinos e cardiovasculares (3, 12, 16, 22-29). Existem também relatos da influência desta condição no ciclo circadiano. A ocorrência destes problemas é maior em função do local, extensão e momento em que ocorre a lesão cerebral $(4,22)$.

Na população com PC podem existir complicações associadas que influenciam as competências alimentares, nomeadamente disfunções oromotoras, refluxo gastroesofágico (RGE), postura incorreta, convulsões e movimentos involuntários, que afetam quer a mastigação e a deglutição, quer a autoalimentação (12, 16, 24, $28,30,31)$. Também as dificuldades auditivas, visuais e cognitivas dificultam a comunicação, o que prejudica a alimentação (32). É de salientar que estas dificuldades se agravam com um maior comprometimento motor, o que justifica o amplo uso de tecnologia assistida e a necessidade de auxílio na alimentação (12, 21, 26, 28 , 30, 32-34).

As dificuldades alimentares, de uma forma geral, manifestam-se precocemente, são comuns e graves. Estas condicionam a capacidade alimentar, o crescimento, o estado nutricional e por conseguinte, a saúde e a QV (3, 4, 12, 16, 21, 24, 28, 33, 35-38). Fatores como ingestão energética, atividade física, tónus muscular, dificuldades cognitivas e dores musculares podem ter influência no estado nutricional, conduzindo à desnutrição ou ao excesso de peso/obesidade $(3,16$, $23,26,28,30,33,36,37)$. Por outro lado, os cuidadores tendem a compensar as dificuldades oferecendo uma elevada quantidade de alimentos, principalmente em crianças alimentadas via gastrostomia $(12,24,27,29,33)$.

Para assegurar um bom estado nutricional, a população pediátrica com PC necessita de uma identificação precoce das dificuldades alimentares e motoras, além de cuidados multidisciplinares complexos, nos quais o acompanhamento nutricional individualizado com o uso dos padrões de referência adequados é essencial (3, 4, $16,27,29,33)$

\section{OBJETIVOS}

Foi objetivo deste trabalho realizar uma revisão sistemática sobre as dificuldades alimentares em crianças e adolescentes com PC e o seu impacto negativo na saúde e QV.

\section{METODOLOGIA}

Foi efetuada uma pesquisa de artigos científicos, segundo a metodologia PRISMA, nas bases de dados online PubMed e Scielo com a expressão de pesquisa: (((cerebral palsy)) AND ((feeding) OR (feeding problems) OR (feeding difficulties) OR (feeding disorders))) AND ((nutrition) OR (nutrition intervention) OR (nutritional intervention) OR (dietary intervention)), até maio de 2020, sem restrição temporal da publicação e de idioma dos artigos (Tabela 1). Na PubMed, foram adicionados os filtros: "Free fulltext", "Human" e "Age" (Newborn, Infant, Preschool Child, Child, Adolescent). A aplicação do filtro "Free fulltext" poderá ter criado restrições no que diz respeito às fontes de informação, nomeadamente quanto à disponibilização de dados. Na Scielo não foram adicionados filtros.

Foram tomadas medidas para minimizar o viés no processo de seleção dos estudos. Contudo, não foram utilizados métodos adicionais para a identificação de artigos relevantes, como por exemplo, pesquisa manual e por referências. Da análise e revisão do resultado das pesquisas, uma amostra total de 19 artigos foi considerada para a constituição deste trabalho (Figura 1).

Para a constituição dos desenhos dos estudos selecionados consistiram em: descritivo, transversal, de coorte, prospetivo e longitudinal. Foram excluídos estudos publicados no formato de revisões de literatura e estudos qualitativos. Os conteúdos descritivos de cada estudo em análise foram: tipo de estudo, características da amostra, instrumentos de avaliação e principais resultados relativos às dificuldades alimentares em crianças e adolescentes com PC (Tabela 2).

O processo de pesquisa, seleção, análise e avaliação do risco de viés foi realizado pelas duas investigadoras de forma independente. Quando se verificou ausência de concordância, foram analisadas as respetivas interpretações e concluída a análise conjunta.

Não foi possível realizar uma síntese quantitativa dos resultados, devido à heterogeneidade clínica dos artigos considerados, pelo que a abordagem narrativa foi a metodologia seguida. 
Tabela 1

Termos de Pesquisa

\begin{tabular}{lcc}
\multicolumn{1}{c}{ PALAVRA-CHAVE } & RESULTADOS POR PALAVRA-CHAVE & RESULTADOS POR GRUPO \\
DE PALAVRA-CHAVE & 30084 \\
\hline "cerebral palsy" & 30084 & \\
\hline "feeding" & 301481 & 314897 \\
\hline "feeding problems" & 14362 & \\
\hline "feeding difficulties" & 6274 & \\
\hline "feeding disorders" & 45400 & 709824 \\
\hline "nutrition" & 596953 & \\
\hline "nutrition intervention" & 168006 & \\
\hline "nutritional intervention" & 161143 \\
\hline "dietary intervention" & 223570 \\
\hline
\end{tabular}

\section{Figura 1}

Termos de Pesquisa

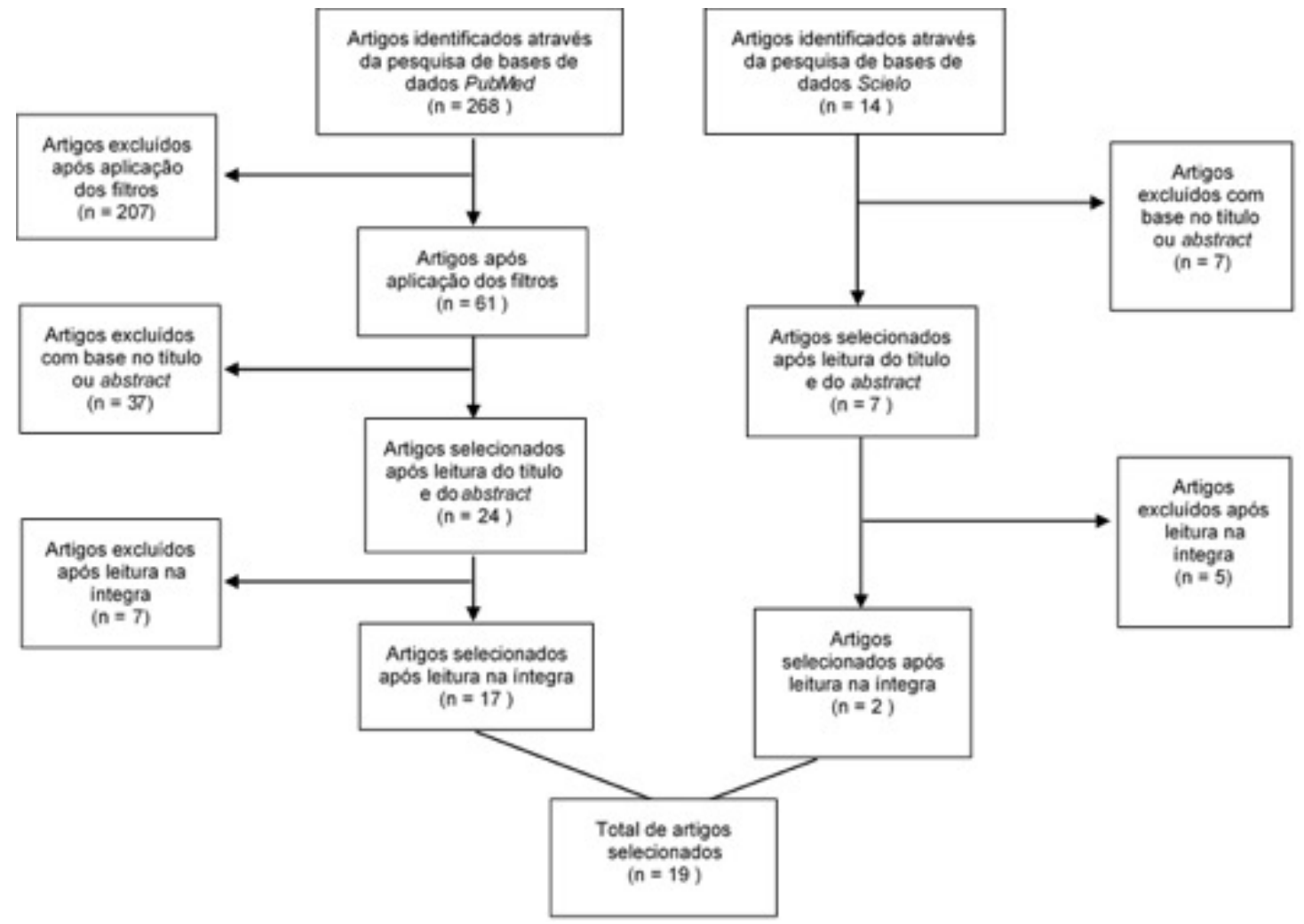

Tabela 2

Tabela de artigos analisados na revisão sistemática

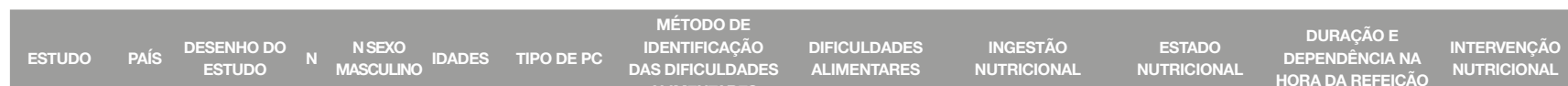

\begin{tabular}{|c|c|c|c|c|c|c|c|c|c|c|c|c|}
\hline $\begin{array}{l}\text { Sullivan et al. } \\
\text { (2000) }\end{array}$ & Inglaterra & Transversal & 271 & NR & $4 A-13 A$ & $\begin{array}{l}\text { Tetraplegia } \\
\text { espástica } \\
\text { Hemiplegia } \\
\text { Discinética } \\
\text { Atáxica } \\
\text { Não classificável }\end{array}$ & $\begin{array}{l}\text { Questionário } \\
\text { destinado aos pais }\end{array}$ & $\begin{array}{l}28 \% \text { sialorreia } \\
59 \% \text { obstipação } \\
22 \% \text { vómitos } \\
\text { frequentes }\end{array}$ & $\begin{array}{l}8 \% \text { recebiam } \\
\text { suplementos } \\
\text { nutricionais } \\
8 \% \text { eram alimentados } \\
\text { via gastrostomia }\end{array}$ & $\begin{array}{l}64 \% \text { nunca foi } \\
\text { avaliado }\end{array}$ & $\begin{array}{l}89 \% \text { necessidade de } \\
\text { auxilio } \\
56 \% \text { engasgava-se } \\
\text { com os alimentos } \\
20 \% \text { dos pais } \\
\text { consideraram a } \\
\text { alimentação difícil e } \\
28 \% \text { demorada }\end{array}$ & NA \\
\hline $\begin{array}{l}\text { Vik et al. } \\
\text { (2001) }\end{array}$ & Noruega & Transversal & 154 & NR & $3 A-19 A$ & $\begin{array}{l}\text { Diplegia } \\
\text { espástica, } \\
\text { Hemiplegia } \\
\text { espástica, } \\
\text { Tetraplegia } \\
\text { espástica } \\
\text { Mista }\end{array}$ & $\begin{array}{l}\text { Questionário } \\
\text { destinado aos pais }\end{array}$ & $\begin{array}{l}26 \% \text { disfunção } \\
\text { oromotora } \\
6 \% \text { disfunção } \\
\text { oromotora grave }\end{array}$ & $\begin{array}{l}9 \% \text { dos pais relataram } \\
\text { pouco apetite da } \\
\text { criança } \\
18 \% \text { baixa ingestão } \\
\text { alimentar dos filhos } \\
12 \% \text { elevada ingestão } \\
\text { alimentar dos filhos }\end{array}$ & $\begin{array}{l}29 \% \text { baixa estatura } \\
10 \% \text { baixo peso } \\
7 \% \text { excesso de peso } \\
60 \% \\
\text { normoponderal }\end{array}$ & $\begin{array}{l}64 \% \text { completamente } \\
\text { independentes na } \\
\text { hora das refeições } \\
33 \% \text { dependiam } \\
\text { de terceiros para se } \\
\text { alimentar } \\
\text { Crianças com } \\
\text { tetraplegia } \\
\text { despenderam mais } \\
\text { tempo em todas as } \\
\text { refeições do que as } \\
\text { outras crianças } \\
(p<0,05)\end{array}$ & NA \\
\hline
\end{tabular}


Tabela 2

Continuação

\begin{tabular}{|c|c|c|c|c|c|c|c|c|c|c|c|}
\hline ESTUDO & PAís & $\begin{array}{l}\text { DESENHO DO } \\
\text { ESTUDO }\end{array}$ & $\mathrm{N}$ & $\begin{array}{l}\text { NSEXO } \\
\text { MASCULINO IDADES }\end{array}$ & TIPO DE PC & $\begin{array}{l}\text { MÉTODO DE } \\
\text { IDENTIFICAÇÃ̄O } \\
\text { DAS DIFICULDADES } \\
\text { ALIMENTARES }\end{array}$ & $\begin{array}{l}\text { DIFICULDADES } \\
\text { ALIMENTARES }\end{array}$ & $\begin{array}{c}\text { INGESTÃO } \\
\text { NUTRICIONAL }\end{array}$ & $\begin{array}{l}\text { ESTADO } \\
\text { NUTRICIONAL }\end{array}$ & $\begin{array}{c}\text { DURAÇÃO E } \\
\text { DEEPENDÊNCIA NA } \\
\text { HORA DA REFEIÇÃOO }\end{array}$ & $\begin{array}{l}\text { INTERVENÇÃO } \\
\text { NUTRICIONAL }\end{array}$ \\
\hline
\end{tabular}

\begin{tabular}{|c|c|c|c|c|c|c|c|c|c|}
\hline $\begin{array}{l}\text { Troughton } \\
\text { et al. } \\
\text { (2001) }\end{array}$ & Irlanda & Transversal & 90 & 56 & $2 A-18 A$ & $\begin{array}{l}\text { Diplegia } \\
\text { Tetraplegia } \\
\text { Hemiplegia } \\
\text { Discinética }\end{array}$ & $\begin{array}{l}\text { Multidisciplinary } \\
\text { Feeding Profile }\end{array}$ & $\mathrm{NE}$ & $\begin{array}{l}3 \% \text { eram alimentadas } \\
\text { via } \\
\text { gastrostomia }\end{array}$ \\
\hline $\begin{array}{l}\text { Motion et al. } \\
\text { (2002) }\end{array}$ & Inglaterra & $\begin{array}{c}\text { Coorte } \\
\text { de base } \\
\text { populacional }\end{array}$ & $33^{a}$ & NR & NR & $\mathrm{NE}$ & $\begin{array}{l}\text { Standard Recording of } \\
\text { Central Motor Deficit } \\
\text { Questionário } \\
\text { destinado ao médico } \\
\text { da escola }\end{array}$ & $\begin{array}{l}\text { Aos } 4 \text { anos de } \\
\text { idade, } 46 \% \text { tinha } \\
\text { dificuldades para } \\
\text { engolir e } 48 \% \\
\text { sialorreia. } \\
\text { Aos } 8 \text { anos de idade, } \\
18 \% \text { tinha dificuldades } \\
\text { para engolir e } 33 \% \\
\text { sialorreia }\end{array}$ & $\begin{array}{l}3 \% \text { eram alimentadas } \\
\text { via gastrostomia }\end{array}$ \\
\hline
\end{tabular}

\begin{tabular}{|c|c|c|c|c|c|c|c|c|c|c|c|c|}
\hline $\begin{array}{l}\text { Sullivan et al. } \\
\text { (2002) }\end{array}$ & Inglaterra & Transversal & 100 & 60 & $4 A-14 A$ & NE & $\begin{array}{l}\text { Questionário } \\
\text { destinado aos pais } \\
\text { Visita domiciliária de } \\
\text { um nutricionista }\end{array}$ & NR & $\begin{array}{l}9 \% \text { com deficiências } \\
\text { graves eram } \\
\text { alimentadas via } \\
\text { gastrostomia } \\
20 \% \text { atingiam um } \\
\text { consumo energético } \\
\text { acima da EAR para } \\
\text { a idade. }\end{array}$ & $\begin{array}{l}\text { abaixo do peso } \\
\text { médio para a sua } \\
\text { idade e sexo } \\
\text { O peso para a idade, } \\
\text { ficou abaixo da faixa } \\
\text { de referência em } \\
8,16 \text { e } 49 \% \text { dos } \\
\text { participantes para os } \\
\text { grupos de deficieincia } \\
\text { leve, moderada e } \\
\text { grave, respetivamente } \\
39 \% \text { dos pais } \\
\text { consideraram que } \\
\text { o seu filho estava } \\
\text { abaixo do peso }\end{array}$ & $\begin{array}{l}48 \% \text { das chanças } \\
\text { com um grave } \\
\text { comprometimento } \\
\text { motor não se } \\
\text { conseguiram alimentar } \\
\text { sozinhas } \\
\text { A totalidade das } \\
\text { crianças com grau } \\
\text { leve a moderado } \\
\text { eram alimentadas por } \\
\text { via oral enenhuma } \\
\text { precisou "sempre" de } \\
\text { assistência na } \\
\text { alimentação }\end{array}$ & NA \\
\hline $\begin{array}{l}\text { Hung et al. } \\
\text { (2003) }\end{array}$ & Tailândia & Transversal & 75 & 47 & $5 \mathrm{M}-10 \mathrm{~A}$ & Espástica & Entrevista aos pais & 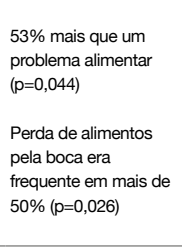 & NR & $\begin{array}{l}41 \% \text { abaixo } \\
\text { do percentil } 10 \\
\text {-desnutrição } \\
4 \% \text { acima do percentil } \\
90 \text {-obesidade }\end{array}$ & $\begin{array}{l}52 \% \text { dependiam } \\
\text { totalmente de } \\
\text { terceiros na } \\
\text { alimentação } \\
48 \% \text { dependiam } \\
\text { parcialmente ou } \\
\text { eram independentes } \\
\text { no processo de } \\
\text { alimentação }\end{array}$ & NA \\
\hline $\begin{array}{l}\text { Craig et al. } \\
(2006)\end{array}$ & Inglaterra & $\begin{array}{l}\text { Prospetivo } \\
\text { controlado }\end{array}$ & 76 & 44 & $4 \mathrm{M}-17 \mathrm{~A}$ & NE & $\begin{array}{l}\text { Questionário } \\
\text { estruturado com } \\
\text { histórico médico } \\
\text { e exame por um } \\
\text { pediatra estagiário } \\
\text { Videofluoroscopia }\end{array}$ & $\begin{array}{l}78 \% \text { diagnosticadas } \\
\text { com problemas } \\
\text { oromotores } \\
69 \% \text { aspiração } \\
\text { orofaringea } \\
47 \% \text { obstipação } \\
75 \% \text { RGE }\end{array}$ & $\begin{array}{l}65 \% \text { recebiam } \\
\text { alimentação } \\
\text { nasogástrica } \\
\text { No pré-operatório: } \\
53 \% \text { das crianças } \\
\text { alcançaram menos de } \\
2 / 3 \text { da EAR; } \\
7 \% \text { atingiram a EAR } \\
\text { recomendada } \\
\text { No pós-operatório } \\
47 \% \text { atingiam menos } \\
2 / 3 \text { da EAR para energia } \\
7 \% \text { alcançavam a } \\
\text { EAR recomendada }\end{array}$ & $\begin{array}{l}\text { O peso antes da } \\
\text { cirurgia variou de } 6 \\
\text { a 33kg } \\
\text { Pré-gastrostomia: } \\
\text { peso médio para a } \\
\text { idade era de } 2,41 \sigma \\
\text { Pós-gastrostomia: } \\
\text { peso médio para a } \\
\text { idade era de } 2,92 \sigma \\
\text { Aumento significativo } \\
\text { na velocidade de } \\
\text { crescimento }\end{array}$ & NR & $\begin{array}{l}\text { Gastrostomia (com } \\
\text { posterior } \\
\text { redução dos } \\
\text { episódios } \\
\text { de sialorreia, } \\
\text { secreções, vómitos } \\
\text { e obstipação) }\end{array}$ \\
\hline $\begin{array}{l}\text { Campos et al. } \\
\text { (2007) }\end{array}$ & Portugal & Transversal & 105 & 63 & $4 \mathrm{~A}-12 \mathrm{~A}$ & NE & FFA & $\begin{array}{l}48 \% \text { capacidade } \\
\text { alimentar moderada } \\
\text { a grave }\end{array}$ & NR & $45 \%$ desnutrição & NR & NA \\
\hline $\begin{array}{l}\text { Somerville et } \\
\text { al. (2008) }\end{array}$ & Austrália & Longitudinal & $249^{b}$ & 173 & $7 \mathrm{M}-19 \mathrm{~A}$ & $\begin{array}{l}\text { Tetraplegia } \\
\text { espástica }\end{array}$ & $\begin{array}{l}\text { Avaliação } \\
\text { multidisciplinar clínica, } \\
\text { com histórico médico } \\
\text { detalhado } \\
\text { Questionário } \\
\text { destinado aos pais/ } \\
\text { cuidadores }\end{array}$ & $\begin{array}{l}92 \% \text { disfagia } \\
67 \% \text { história atual ou } \\
\text { antecedente de RGE } \\
13 \% \text { hematémeses }\end{array}$ & NR & $\begin{array}{l}72 \% \text { crescimento } \\
\text { insuficiente ou baixa } \\
\text { estatura }\end{array}$ & $\begin{array}{l}92 \% \text { totalmente } \\
\text { dependentes dos } \\
\text { cuidadores para } \\
\text { alimentação }\end{array}$ & $\begin{array}{l}\text { Aconselhamento } \\
\text { nutricional, } \\
\text { adequação da } \\
\text { alimentação e } \\
\text { informações } \\
\text { sobre controlo } \\
\text { salivar e higiene } \\
\text { oral (gastrostomia } \\
\text { recomendada } \\
\text { a } 140 \text { crianças, } \\
\text { realizada em } 91 \\
\text { e fundoplicatura } \\
\text { recomendada a 111, } \\
\text { realizada em 74) }\end{array}$ \\
\hline $\begin{array}{l}\text { Silvério et al. } \\
\text { (2009) }\end{array}$ & Brasil & Longitudinal & 36 & 23 & $1 \mathrm{~A}-7 \mathrm{~A}$ & $\begin{array}{l}\text { Tetraplegia } \\
\text { espástica }\end{array}$ & $\begin{array}{l}\text { FOIS } \\
\text { Protocolo ROGS } \\
\text { Verificação das } \\
\text { consistências } \\
\text { alimentares ingeridas } \\
\text { Verificação da } \\
\text { presença de } \\
\text { sinais sugestivos } \\
\text { de penetração e/ } \\
\text { ou aspiração } \\
\text { laringotraqueal }\end{array}$ & $\begin{array}{l}\text { Pré-intervenção: } \\
61 \% \text { classificados } \\
\text { com nível V da escala } \\
\text { FOIS ( } p=0,043) \\
\text { Pós-intervenção: } \\
33 \% \text { classificados } \\
\text { com nível V da escala } \\
\text { FOIS ( } p=0,043 \text { ) } \\
50 \% \text { disfagia } \\
\text { orofaríngea leve } \\
(p=0,035) \text { e } 36 \% \text { grave } \\
89 \% \text { hipersecretividade }\end{array}$ & $\begin{array}{l}\text { Pré-intervenção: } \\
80 \% \text { faziam ingestão } \\
\text { de liquidos }(p<0,001) \\
23 \% \text { de liquidos } \\
\text { espessos }(p=0,002) \\
\\
\text { Pós-intervenção: } \\
30 \% \text { faziam ingestão } \\
\text { de liquidos ( } p<0,001) \\
60 \% \text { de líquidos } \\
\text { espessos } \\
(p=0,002)\end{array}$ & NR & NR & $\begin{array}{c}\text { Reabilitação } \\
\text { funcional de } \\
\text { disfagia orofaríngea }\end{array}$ \\
\hline
\end{tabular}




\section{Tabela 2}

Continuação

\begin{tabular}{|c|c|c|c|c|c|c|c|c|c|c|c|}
\hline ESTUDO & PAÍS & $\begin{array}{l}\text { DESENHO DO } \\
\text { ESTUDO }\end{array}$ & $\mathrm{N}$ & $\begin{array}{l}\text { NSEXO } \\
\text { MASCULINO IDADES }\end{array}$ & TIPO DE PC & $\begin{array}{l}\text { MÉTODO DE } \\
\text { IDENTIFICAÇÃO } \\
\text { DAS DIFICULDADES } \\
\text { ALIMENTARES }\end{array}$ & $\begin{array}{l}\text { DIFICULDADES } \\
\text { ALIMENTARES }\end{array}$ & $\begin{array}{l}\text { INGESTÃO } \\
\text { NUTRICIONAL }\end{array}$ & $\begin{array}{l}\text { ESTADO } \\
\text { NUTRICIONAL }\end{array}$ & $\begin{array}{c}\text { DURAÇÃO E } \\
\text { DEPENDÊNCIA NA } \\
\text { HORA DA REFEIÇÃO }\end{array}$ & $\begin{array}{l}\text { INTERVENÇÃO } \\
\text { NUTRICIONAL }\end{array}$ \\
\hline
\end{tabular}

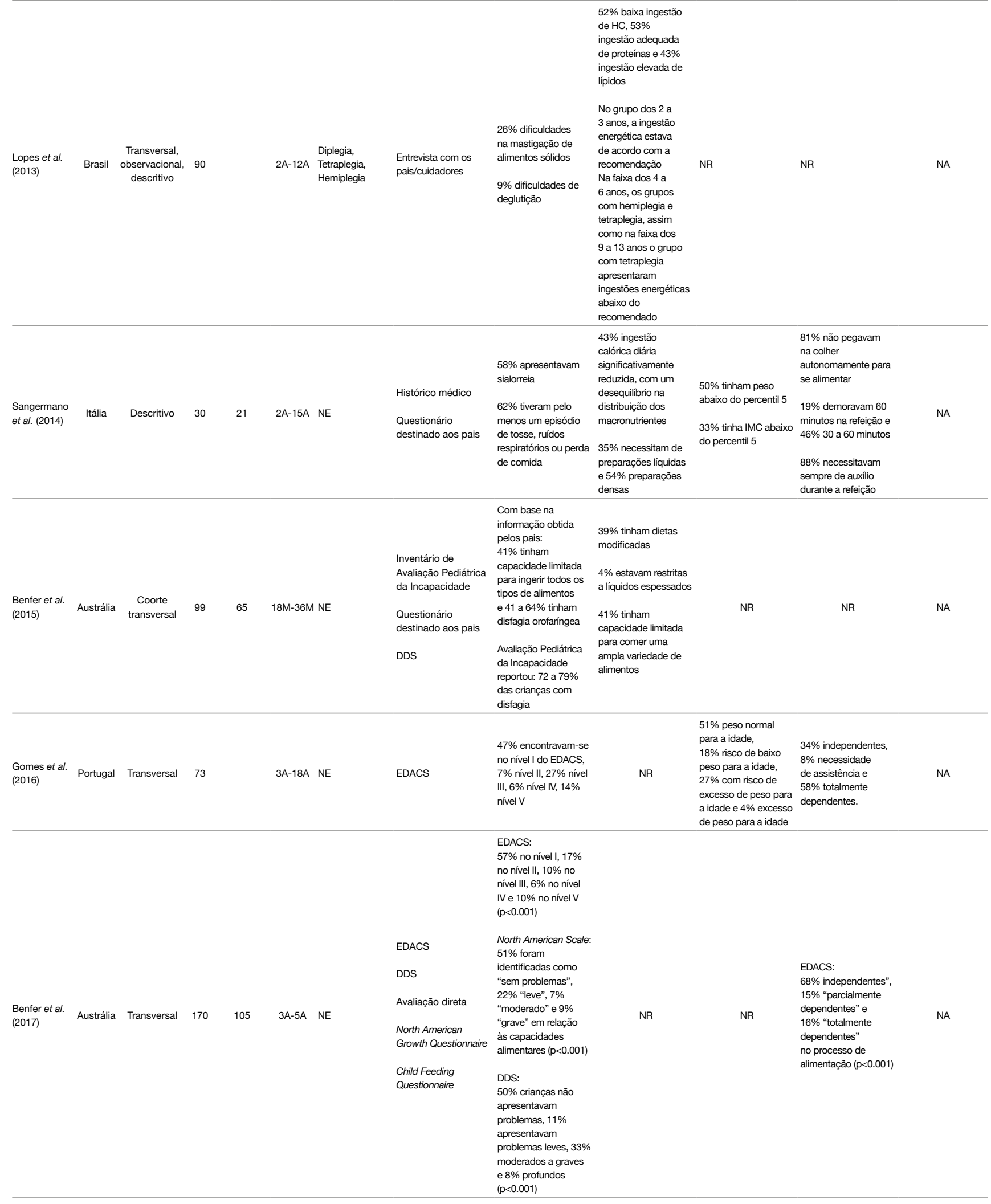


Tabela 2

Continuação

\begin{tabular}{|c|c|c|c|c|c|c|c|c|c|c|c|c|}
\hline ESTUDO & PAís & $\begin{array}{l}\text { DESENHO DO } \\
\text { ESTUDO }\end{array}$ & $\mathrm{N}$ & $\begin{array}{c}\text { NSEXO } \\
\text { MASCULUNO }\end{array}$ & IDADES & TIPO DE PC & $\begin{array}{l}\text { MÉTODO DE } \\
\text { IDENTIFICAÇĀO } \\
\text { DAS DIFICULDADES } \\
\text { ALIMENTARES }\end{array}$ & $\begin{array}{l}\text { DIFICULDADES } \\
\text { ALIMENTARES }\end{array}$ & $\begin{array}{l}\text { INGESTÃO } \\
\text { NUTRICIONAL }\end{array}$ & $\begin{array}{c}\text { ESTADO } \\
\text { NUTRICIONAL }\end{array}$ & $\begin{array}{c}\text { DURAÇÃO E } \\
\text { DEPENDÊNCIA NA } \\
\text { HORA DA REFEIÇÃOO }\end{array}$ & $\begin{array}{l}\text { INTERVENÇÃO } \\
\text { NUTRICIONAL }\end{array}$ \\
\hline $\begin{array}{l}\text { Benfer et al. } \\
\text { (2017) }\end{array}$ & Austrália & $\begin{array}{l}\text { Longitudinal } \\
\text { de base } \\
\text { populacional }\end{array}$ & 179 & 111 & $18 \mathrm{M}-60 \mathrm{M}$ & NE & $\begin{array}{l}\text { DDS } \\
\text { EDACS } \\
\text { Child Feeding } \\
\text { Questionnaire para } \\
\text { PC. }\end{array}$ & $\begin{array}{l}4 \text { grupos de sinais } \\
\text { clinicos, dos quais } \\
3 \text { se relacionaram } \\
\text { significativamente } \\
\text { com as dificuldades } \\
\text { alimentares e de } \\
\text { deglutição: } \\
\text { Grupo 1: sibilância, } \\
\text { estridor, ruídos } \\
\text { respiratórios e } \\
\text { rouquidão ( } p=0,002) \\
\text { Grupo 2: vómito e } \\
\text { recusa alimentar } \\
(p=0,001) \\
\text { Grupo 3: tosse, } \\
\text { engasgo, deglutições } \\
\text { múltiplas e pigarrear } \\
\text { (p<0,001) }\end{array}$ & NR & NR & NR & NA \\
\hline $\begin{array}{l}\text { Caramico- } \\
\text { Favero et al. } \\
\text { (2018) }\end{array}$ & Brasil & Transversal & 40 & 23 & $4 \mathrm{~A}-10 \mathrm{~A}$ & $\begin{array}{l}\text { Tetraparesia } \\
\text { espástica } \\
\text { Discinética não } \\
\text { espástica }\end{array}$ & $\begin{array}{l}\begin{array}{l}\text { Avaliação por } \\
\text { fonoaudiólogos, } \\
\text { gastroenterologista }\end{array} \\
\text { Questionário } \\
\text { destinado aos pais/ } \\
\text { cuidadores }\end{array}$ & $\begin{array}{l}83 \% \text { disfagia } \\
40 \% \text { sintomas } \\
\text { sugestivos de RGE } \\
60 \% \text { obstipação }\end{array}$ & $\begin{array}{l}30 \% \text { consumia menos } \\
\text { de } 90 \% \text { das EER } \\
20 \% \text { consumia entre } \\
91 \text { a } 120 \% \text { do EER } \\
50 \% \text { mais de } 120 \% \\
\text { do EER } \\
\text { Na presença de } \\
\text { disfagia observou-se } \\
\text { uma menor ingestão } \\
\text { energética ( } p=0,009 \text { ), } \\
\text { um maior consumo de } \\
\text { HC ( }(=0,023) \text { e uma } \\
\text { menor ingestão de } \\
\text { liquidos ( } p=0,001) \\
\text { Na presença de RGE, } \\
\text { a ingestão de líquidos } \\
\text { era maior ( } p=0,042) \\
\text { As crianças } \\
\text { com obstipação } \\
\text { apresentavam um } \\
\text { menor consumo diário } \\
\text { de fibras }(p=0,031) \text { e } \\
\text { líquidos }(p=0,013)\end{array}$ & NR & NR & NA \\
\hline $\begin{array}{l}\text { Polack et al. } \\
\text { (2019) }\end{array}$ & Gana & Transversal & 76 & 40 & $18 \mathrm{M}-12 \mathrm{~A}$ & NE & Entrevista aos pais & $\begin{array}{l}50 \% \text { dos pais } \\
\text { referiram problemas } \\
\text { de alimentação pelo } \\
\text { menos "às vezes }\end{array}$ & $\begin{array}{l}50 \% \text { dos pais } \\
\text { estavam "sempre" } \\
\text { preocupados com a } \\
\text { ingestão inadequada } \\
\text { de alimentos }\end{array}$ & $\begin{array}{l}65 \% \text {, abaixo dos } 5 \\
\text { anos, apresentavam } \\
\text { baixo peso } \\
54 \% \text { défice no de } \\
\text { crescimento }\end{array}$ & $\begin{array}{l}75 \% \text { dos cuidadores } \\
\text { relataram que o filho } \\
\text { precisava "sempre" } \\
\text { de assistência com a } \\
\text { alimentação }\end{array}$ & NA \\
\hline $\begin{array}{l}\text { Bell et al. } \\
\text { (2019) }\end{array}$ & Austrália & $\begin{array}{l}\text { Prospetivo, } \\
\text { transversal }\end{array}$ & 89 & 63 & $2 \mathrm{~A}-19 \mathrm{~A}$ & NE & $\begin{array}{l}\text { Avaliação por } \\
\text { fonoudiólogos } \\
\text { pediátricos } \\
\text { DDS } \\
\text { VFSS } \\
\text { EDACS } \\
\begin{array}{l}\text { Questionário } \\
\text { destinado aos pais/ } \\
\text { cuidadores }\end{array}\end{array}$ & $\begin{array}{l}\text { DDS: } \\
67 \% \text { apresentaram } \\
\text { dificuldades } \\
\text { alimentares, } \\
\text { em } 29 \% \text { estas } \\
\text { condicionavam a } \\
\text { segurança alimentar } \\
\\
\text { Questionário: } \\
48 \% \text { tinham risco } \\
\text { de apresentarem } \\
\text { dificuldades } \\
\text { alimentares erisco de } \\
\text { desnutrição }\end{array}$ & NR & $\begin{array}{l}71 \% \text { bem nutridos, } \\
22 \% \text { desnutrição } \\
\text { moderada e } 7 \% \\
\text { desnutrição severa }\end{array}$ & $\begin{array}{l}\text { EDACS: } \\
56 \% \text { independentes, } \\
26 \% \text { necessidade } \\
\text { de assistência, } \\
18 \% \text { totalmente } \\
\text { dependentes }\end{array}$ & $\begin{array}{c}\text { Encaminhadas para } \\
\text { VFSS }\end{array}$ \\
\hline
\end{tabular}

Níveis do EDACS: I - Come e bebe em segurança e de forma eficiente, II - Come e bebe em segurança, mas com alguma limitação na eficiência, III - Come e bebe com alguma limitação na segurança; pode haver limitações na eficiência, IV - Come e bebe com limitações significativas na segurança e V - Incapaz de comer ou beber em segurança, a alimentação por sonda pode ser considerada para assegurar a nutrição

${ }^{a} \mathrm{~N} .{ }^{\circ}$ de crianças diagnosticadas com PC

${ }^{b} \mathrm{~N} .{ }^{\circ}$ de crianças, num estudo que envolveu também adultos

FFA: Functional Feeding Assessment Test

FOIS: Functional Oral Intake Scale

HC: Hidratos de Carbono

IMC: Índice de Massa Corporal

M: Meses

NA: Não aplicável

NE: Não especificado

NR: Não reportado

PC: Paralisia Cerebral

RGE: Refluxo Gastroesofágico

DDS: Dysphagia Disorders Survey

EAR: Estimated Average Requirement

EDACS: Eating and Drinking Ability Classification System

VFSS: Videofluoroscopic Swallow Study

EER: Estimated Energy Requirements 


\section{RESULTADOS}

Segundo Sullivan et al. (2000), da totalidade da amostra, 93\% apresentaram PC, 47\% não conseguiam andar, 78\% possuíam dificuldades na fala e $31 \%$ tinham sofrido pelo menos uma infeção respiratória nos 6 meses anteriores. Este estudo revelou ainda que crianças total ou parcialmente incapazes de andar eram mais propensas a ter problemas de deglutição e engasgos frequentes com sólidos e líquidos ( $p<0,0001)$, precisando de assistência na alimentação. As crianças que se babavam com frequência tinham maior risco de sofrer disfagia, precisar de mais tempo e auxílio na alimentação. Houve também uma correlação significativa entre a sialorreia e o prazer de comer, dado que em $32 \%$ das crianças que se babavam com frequência, os seus cuidadores acreditavam que estas não apreciavam as refeições. As crianças que se engasgavam com líquidos $(p<0,003)$ e que apresentavam dificuldades para engolir alimentos ( $p=0,0002)$ eram mais propensas a ter infeções respiratórias, necessitavam de mais tempo e auxílio na alimentação $(p<0,03)$, assim como, relatavam vómitos frequentes $(p<0,0001)$. Estas também mostravam pouco interesse pela alimentação $(p<0,02)$ e os pais estavam mais preocupados com os seus problemas alimentares $(p<0,04)$. Sullivan et al. (2000) evidenciaram que a alimentação e o estado nutricional de $64 \%$ das crianças nunca foram avaliados, embora $38 \%$ dos pais considerassem que o seu filho tinha baixo peso.

De acordo com os resultados de Vik et al. (2001), as crianças com disfunção oromotora não diferiram das sem disfunção em relação ao apetite, contudo 13 dos 35 pais relataram uma baixa ingestão alimentar dos filhos, em comparação com 8 dos 70 pais de crianças sem disfunção oromotora $(p<0,001)$. Em casos de disfunção oromotora, o tempo gasto na alimentação era superior comparativamente aos casos sem disfunção ( $p<0,001)$. Quanto à procura de ajuda nutricional, 9 em 10 crianças com várias complicações oromotoras já tinham consultado um nutricionista, enquanto apenas 23 das 40 com 1 complicação tiveram esse contacto. Em relação ao impacto no contexto familiar, $15 \%$ dos pais relataram que a sua QV seria superior sem os problemas alimentares.

Troughton et al. (2001) confirmaram uma associação entre o comprometimento dos membros superiores e a função oromotora, uma vez que, crianças com quadriplegia apresentaram scores menores de competência alimentar em comparação com outros grupos $(p<0,001)$. Também observaram uma correlação entre competências alimentares e percentis de peso, pregas cutâneas subescapular e tricipital e circunferência do braço $(p<0,02)$. Este estudo demonstrou que melhores competências de autoalimentação estavam associadas a um estado nutricional adequado $(\mathrm{p}<0,02)$, sendo que, em todos os aspetos da alimentação, os desnutridos apresentaram scores mais baixos que crianças adequadamente nutridas $(p<0,002)$.

Motion et al. (2002) afirmaram que exaustão, asfixia e fraca sucção durante a alimentação às 4 semanas de idade eram frequentes no grupo com PC. Nove em cada 10 crianças com PC e com fraca sucção às 4 semanas de idade não conseguiram andar aos 4 anos, em comparação com 3 das 8 crianças que relataram "mamar" normalmente às 4 semanas $(p=0,009)$. Dificuldades na alimentação aos 6 meses foram relatadas em $10 \%$ do grupo com PC e em 3\% do grupo controlo ( $p=0,017)$. Aos 8 anos, a disfunção oromotora foi menos frequente quando comparada aos 4 anos, tendo havido melhoria na deglutição e no controlo da saliva. Cinco das 9 crianças que necessitavam de adaptações de texturas alimentares aos 8 anos de idade relataram exaustão na alimentação às 4 semanas, em comparação com 5 das 23 que conseguiram ingerir alimentos sem adaptações $(p=0,05)$. Cinco das 10 crianças que estavam abaixo do peso (percentil 0,4 no UK Reference Growth Chart) aos 8 anos demonstraram exaustão durante a alimentação às 4 semanas de idade, em comparação com 5 das 22 que não estavam abaixo do peso $(p=0,01)$. Três das 4 crianças com problemas de alimentação aos 6 meses estavam abaixo do peso aos 8 anos, em comparação com 1 das 4 que não tiveram problemas alimentares precoces $(p=0,013)$. As dificuldades de alimentação às 4 semanas de idade foram associadas ao comprometimento funcional aos 4 anos $(p=0,009)$, estar abaixo do peso $(p=0,01)$ e apresentar dificuldades de fala e deglutição $(p=0,005)$ aos 8 anos de idade.

Hung et al. (2003) observaram uma associação entre a desnutrição e a perda de alimentos pela boca $(p=0,026)$ e apresentarem mais que 1 problema na alimentação $(p=0,044)$. Neste estudo, foi demonstrado que a desnutrição estava associada à dependência do cuidador para alimentação $(p<0,001)$.

No estudo de Campos et al. (2007), as capacidades alimentares diferiam entre crianças desnutridas e não desnutridas $(p<0,001)$. 0 peso e as pregas cutâneas subescapular e tricipital relacionaram-se com as competências alimentares, sendo a correlação mais forte entre o peso e o parâmetro "beber pela chávena" ( $p=0,001)$.

Sangermano et al. (2014) referiram que na amostra, 39\% das crianças tinham historial de doenças respiratórias. Já Somerville et al. (2008) verificaram em $65 \%$ da amostra infeções respiratórias recorrentes ou crónicas. A videofluoroscopia esofágica evidenciou aspiração traqueal em $41 \%$ dos resultados e a endoscopia digestiva alta detetou anormalidades em 57\%, incluindo esofagite (45\%), esófago de Barrett (8\%) e gastrite por Helicobacter pylori (12\%). Os autores reconheceram que pacientes com deficiência exibiram uma ingestão oral reduzida, desnutrição e problemas gastrointestinais que podem conduzir a esofagite crónica e doenças pulmonares.

Silvério et al. (2009) estudaram a reabilitação funcional de disfagia orofaríngea em crianças com PC tetraplégica espástica e observaram que, antes da terapia, $61 \%$ das crianças alimentavam-se via oral, ingeriam múltiplas consistências e necessitavam de uma preparação especial (nível V da escala Functional Oral Intake Scale, $p=0,043$ ), valor que reduziu para 33\% após a intervenção ( $p=0,043)$.

Lopes et al. (2013) observaram que crianças com diplegia não apresentaram dificuldades para mastigar e deglutir, enquanto que na tetraplegia existiram mais dificuldades para mastigar (41\%) e deglutir $(12,8 \%)$, comparadas a 15 e $7 \%$, respetivamente, das crianças com hemiplegia. Este estudo reportou ainda que, a maioria das crianças com PC apresentaram trânsito intestinal regular, já nos restantes grupos era menor que três vezes por semana, em dias alternados. No estudo de Benfer et al. (2015), as crianças que estavam restritas a líquidos espessados foram classificadas no nível V do Gross Motor Function Classification System (GMFCS) - transportado por cadeira de rodas manual. Os autores verificaram uma discrepância entre a informação obtida pelos pais e a recomendação médica de segurança para engolir, o que pode indicar que os pais não identificaram corretamente "capacidade limitada" para determinada textura, apesar de terem identificado com precisão texturas de alimentos para as quais o filho pode precisar de adaptações. Apenas 3 crianças que recebiam alimentos por via oral foram consideradas inseguras em todas as texturas.

Gomes et al. (2016) reconheceram que, quanto maior a gravidade motora e as dificuldades alimentares, maior o risco da criança apresentar baixo peso para a idade e menor o risco de excesso de peso para a idade $(\mathrm{p}<0,05)$.

Benfer et al. (2017) relacionaram a função motora e as competências alimentares, concluindo que a grande percentagem das crianças dos níveis I - Anda sem limitações e II - Anda com limitações do GMFCS 
correspondiam ao nível I - Come e bebe em segurança e de forma eficiente do EDACS $(p<0,001)$ e que a maioria das crianças de nível $\checkmark$ do GMFCS apresentava nível $V$ - Incapaz de comer ou beber em segurança, a alimentação por sonda pode ser considerada para assegurar a nutrição na escala EDACS ( $p<0,001)$.

Benfer et al. (2017) encontraram uma relação entre tosse, engasgos, cerrar os dentes durante a refeição, rouquidão, ruídos respiratórios e o resultado geral da alimentação $(p<0,001)$. Os autores verificaram ainda uma relação entre o relatório dos pais sobre a "dificuldade de comer e beber" dos seus filhos e a desnutrição moderada, no que diz respeito ao Índice de Massa Corporal e ao peso $(p<0,01)$.

Os resultados de Polack et al. (2018) revelaram que $2 / 3$ dos cuidadores relataram estar "sempre" preocupados com a alimentação dos filhos. Os autores observaram ainda que as dificuldades alimentares das crianças foram comuns e estavam associadas ao baixo peso da criança e à pior $Q V$ do cuidador ( $p<0,001)$.

Bell et al. (2019) observaram em 11 crianças sinais sugestivos de aspiração, em mais que uma situação, sendo que as que demonstraram 1 sinal em várias ocasiões, ou mais que 1 sinal diferente numa ou mais ocasiões, foram encaminhadas para videofluoroscopia da deglutição (VFSS). Contudo, apenas em 6 crianças foi obtido o consentimento dos pais, sendo confirmada a aspiração em 4, predominantemente em líquidos finos $(n=3)$, fluídos levemente espessados $(n=2)$, purés $(n=2)$ e todas as texturas $(n=1)$. Após a VFSS, foi recomendado a 1 criança a alimentação via gastrostomia e a 5 crianças modificações de texturas alimentares.

\section{DISCUSSÃO DOS RESULTADOS Dificuldades Alimentares}

As dificuldades alimentares em crianças e jovens com PC são frequentes, podendo ser oromotoras, onde se incluem dificuldades de deglutição e/ou mastigação, como sucção fraca, sialorreia, engasgos frequentes, tosse, perda de alimentos e disfagia que, como consequência, pode acarretar aspiração, que agravando pode conduzir a doenças pulmonares $(26,39-48)$. Problemas gastrointestinais como vómitos e RGE, muitas vezes na origem de esofagite, gastrite e outras doenças no trato gastrointestinal, também se incluem nas complicações alimentares frequentes nesta população, tal como a obstipação (3, 26, 40, 42, 46, 47). Além destes, podem ser comuns a necessidade de assistência durante as refeições e a recusa alimentar (28, 42-45, 47-50).

Os autores, ao aplicarem diversas metodologias para avaliar as competências alimentares, evidenciaram uma elevada prevalência de dificuldades na população pediátrica com PC e afirmaram que a maioria dos indivíduos avaliados se encontrava em níveis mais graves, revelando competências alimentares diminutas e dependência na alimentação $(48,51)$. Por sua vez, o comprometimento da ingestão oral pode levar à necessidade de utilização de outras vias de alimentação, destacando-se a gastrostomia como um procedimento comum nesta população $(41,42,45,50)$.

\section{Ingestão Alimentar}

Somerville et al. (2008) reconheceram que pacientes com dificuldades alimentares apresentaram uma ingestão oral reduzida. Vik et al. (2001), através dos relatos de pais, verificaram a mesma associação, ao observar que a maioria dos pais que consideraram que o seu filho come muito pouco são pais de crianças com disfunção motora oral. No que se refere à energia, foram vários os estudos que confirmaram uma ingestão média significativamente baixa entre as crianças que possuíam alguma dificuldade alimentar (26, 44, 46). Lopes et al. (2013) acrescentaram que, quanto maior o grau de comprometimento motor, menor a ingestão energética.

Já Caramico-Favero et al. (2018) relataram que crianças com disfagia apresentaram maior consumo de $\mathrm{HC}$ quando comparadas a crianças sem disfagia. Os autores avaliaram ainda a ingestão de líquidos e depararam-se com uma ingestão significativamente menor entre as crianças com disfagia e com obstipação. No entanto, nas crianças com RGE a ingestão de líquidos foi maior, comparativamente a crianças sem esta condição.

Segundo Benfer et al. (2015) menos de 50\% das crianças avaliadas tinham limitações na capacidade de ingerir todos os tipos de alimentos, o que realçou a necessidade de modificações nas texturas ou exclusão de algum tipo de alimento das dietas das crianças com PC dos 18 aos 36 meses, contudo estas adaptações foram pouco relatadas pelos pais. Em estudos que consideraram idades mais avançadas (dos 2 aos 19 anos) também foram prescritas texturas modificadas para sólidos e líquidos $(41,45)$.

\section{Estado Nutricional}

A desnutrição é frequente quando nos referimos a crianças e jovens com PC $(28,40,44,45,52,53)$. Este problema é, na maioria dos casos, resultado das dificuldades alimentares $(3,40)$.

Campos et al. (2007) nos seus resultados demonstraram que crianças desnutridas tinham uma capacidade alimentar reduzida, comparativamente a crianças não desnutridas. No mesmo estudo, verificaram uma correlação entre medidas antropométricas e competências alimentares. Outros autores confirmaram esta relação ao observarem que, à medida que as dificuldades alimentares em crianças com graves deficiências neuromotoras aumentam, aumenta o risco de baixo peso e baixa estatura para a idade $(28,43,44,49,53,54)$. Benfer et al. (2017) encontraram a mesma relação, baseada nas "dificuldades de comer e beber" relatadas pelos pais. Os autores Motion et al. (2002) acrescentaram que o baixo peso na criança estava associado a problemas alimentares que ocorriam quando lactentes.

\section{Função Motora Global}

Benfer et al. (2015) verificaram uma maior percentagem de dietas modificadas no grupo de crianças com pior função motora global, sendo que a totalidade das crianças que só conseguiam beber líquidos espessados se encontravam no nível V do GMFCS. Outros estudos confirmaram esta premissa, ao revelarem que crianças mais afetadas em termos motores eram mais propensas a ter dificuldades alimentares $(42,43,50,53)$. Benfer et al. (2017) relataram uma relação evidente entre níveis do GMFCS e do EDACS, em que pior função motora se traduzia em piores competências alimentares.

Quando nos referimos ao tipo de PC, a discrepância em termos de dificuldades alimentares também é clara, como concluiu Lopes et al. (2013) quando verificaram que crianças com tetraplegia, ao contrário das com diplegia, apresentavam mais dificuldades para mastigar e deglutir, comparadas a crianças com hemiplegia. Os resultados de Motion et al. (2002) acrescentaram que, dificuldades alimentares em lactentes estavam associadas ao comprometimento funcional em idades posteriores.

\section{Duração e Dependência na Hora da Refeição}

Vários autores demonstraram que esta população dependia total ou parcialmente de terceiros para o processo de alimentação (48-50) e que o tempo despendido era prolongado, sendo menor em crianças que não apresentaram qualquer comprometimento alimentar (28, 42-44). Sullivan et al. (2000) demonstraram que as crianças que se babavam continuamente, por norma, precisavam de mais tempo e ajuda nas refeições, o que se relacionou inversamente com o prazer alimentar. 
Também foi evidenciado que crianças que se engasgavam com líquidos necessitavam de mais ajuda e maior tempo com a refeição $(47,54)$.

\section{Qualidade de Vida}

Nos estudos de Sullivan et al. (2000) e Polack et al. (2018) a maioria dos pais/cuidadores consideraram a alimentação exaustiva, difícil e demorada, demonstrando que as complicações alimentares das crianças afetam a QV da família. Assim, deve ser dada atenção ao estado emocional dos cuidadores, pois a exaustão pode complicar o processo de alimentação, como demonstra a literatura $(12,21,26$, $28,30,32-34,43)$. Somerville et al. (2008) realçaram os problemas médicos associados a estas complicações que, em conjunto com outras despesas de saúde e educação, têm impacto significativo na economia familiar $(12,55)$.

\section{Procura de Ajuda Nutricional}

Sullivan et al. (2000) referiram que embora alguns pais tenham considerado que o seu filho apresentava baixo peso, mais de metade nunca os submeteram a uma avaliação da alimentação e/ou estado nutricional. Vik et al. (2001) acrescentaram que a procura é maior quando existe um maior número de complicações oromotoras. Isto salienta que, recorrer a ajuda especializada em nutrição não é frequente e depende da gravidade dos casos, muitas vezes devido ao facto de certas complicações não serem facilmente identificadas pelos pais ou mesmo pelo não reconhecimento do papel fundamental do nutricionista destes distúrbios.

\section{Comparação Crítica com a Literatura}

Os resultados desta revisão sistemática vão de encontro aos observados pelos autores Speyer et al. (2019) na única revisão sistemática e meta-análises nesta temática, nomeadamente, em termos da elevada prevalência de problemas de salivação, deglutição e alimentação na PC. Estes autores verificaram ainda uma relação entre estas dificuldades alimentares e um alto risco de desnutrição, doenças pulmonares por aspiração e, subsequentemente, baixa qualidade de vida. Já os autores Menezes et al. (2017) e Novak et al. (2020), em revisões sistemáticas no âmbito de outras temáticas que envolvem a população pediátrica com PC, incluíram estudos que relatam uma elevada frequência de problemas alimentares nesta população, nomeadamente a disfagia, e o seu impacto no estado nutricional e QV.

\section{Limitações}

Através da avaliação da qualidade dos estudos incluídos foram encontrados potenciais riscos de viés que foram considerados na discussão e nas conclusões desta revisão. Importa destacar o tamanho limitado e a heterogeneidade da amostra em alguns dos estudos selecionados, particularmente em termos de grau de PC, nacionalidade e idades. $O$ facto de existir uma variedade de ferramentas para analisar as competências alimentares e o estado nutricional também deve ser considerado. O próprio conceito de "dificuldade alimentar" não é uniforme dentro dos resultados, podendo ser mais ou menos abrangente. Houve, também, uma variação considerável dentro dos métodos de identificação das dificuldades alimentares, destacando-se dados obtidos por registos médicos e questionários aos pais/cuidadores, como métodos mais subjetivos.

\section{CONCLUSÕES}

São inúmeras as possíveis dificuldades alimentares em crianças e adolescentes com PC. Apesar desta população não apresentar um crescimento linear e possuir alterações na sua composição corporal, a procura de um nutricionista ainda é pouco frequente. Contudo, este deve ser consultado assim que surjam dificuldades alimentares, para definir uma terapêutica nutricional individual e adequada a esta população. Só deste modo é possível minimizar as dificuldades e as consequências que delas advêm para melhorar o estado nutricional e a QV.

São necessários mais estudos com metodologias rigorosas nesta temática, nomeadamente, a uniformização das ferramentas para avaliação das competências alimentares, com recurso às mais consensuais na prática clínica e na comunidade científica. É ainda importante que englobem uma faixa etária mais abrangente, uma vez que a literatura relata um aumento da esperança de vida, e por conseguinte, uma maior população adulta com PC e com um número elevado de indivíduos com deficiências graves. Neste sentido, não só permitiria obter um levantamento das competências alimentares na PC de forma mais correta e precisa, como também orientar as intervenções clínicas, tornando-as mais eficazes.

\section{REFERÊNCIAS BIBLIOGRÁFICAS}

1. Rosenbaum P, Paneth N, Leviton A, Goldstein M, Bax M. A Report: The Definition and Classification of Cerebral Palsy. April 2006. Developmental medicine \& child neurology. 2007;49(2):8-14

2. Oskoui M, Coutinho F, Dykeman J, Jetté N, Pringsheim T. An update on the prevalence of cerebral palsy: a systematic review and meta-analysis. Developmental Medicine \& Child Neurology. 2013 Jun 1;55(6):509-19.

3. Lopes PAC, Amancio OMS, Araújo RFC, Vitalle MS de S, Braga JAP. Food pattern and nutritional status of children with cerebral palsy. Revista Paulista de Pediatria. 2013;31(3):344-9.

4. Novak I, Hines M, Goldsmith S, Barclay R. Clinical prognostic messages from a systematic review on cerebral palsy. Pediatrics. 2012;130(5):1285-312.

5. Jarvis S, Glinianaia S, Arnaud C, Fauconnier J, Johnson A, McManus V, et al. Case gender and severity in cerebral palsy varies with intrauterine growth. Archives of Disease in Childhood. 2005;90(5):474-9.

6. Jacobsson B, Hagberg G. Antenatal risk factors for cerebral palsy. Best Practice and Research: Clinical Obstetrics and Gynaecology. 2004 Jun 1;18(3):425-36.

7. Murphy N, Such-Neibar T. Cerebral palsy diagnosis and management: the state of the art. Current Problems in Pediatric and Adolescent Health Care. 2003;33(5):146-69. 8. Centers for Disease Control and Prevention. Data and Statistics for Cerebral Palsy CDC [Internet]. [cited 2020 Apr 22]. Available from: https://www.cdc.gov/ncbddd/cp/data.html. 9. Graham HK. Classifying cerebral palsy. Journal of Pediatric Orthopaedics. 2005;25(1):127-8.

10. Rosa GKB, Marques I, Medina-Papst J, Gobbi LTB. Desenvolvimento motor de criança com paralisia cerebral: avaliação e intervenção. Revista Brasileira De Educação Especial. 2008;14(2):163-76.

11. Lai C, Chen C, Chen C. Longitudinal changes in health-related quality of life in preschool children with cerebral palsy of different levels of motor severity. Research in Developmental Disabilities. 2017;61(5):11-8.

12. Bell K, Boyd R, Tweedy S, Weir K, Stevenson R, Davies P. A prospective, longitudinal study of growth, nutrition and sedentary behaviour in young children with cerebral palsy. BMC Public Health. 2010;10(159):1-12.

13. Virella D, Folha T, Andrada M, Cadete A, Gouveia R, Gaia T, et al. Paralisia Cerebral em Portugal no século XXI. Federação das Associações Portuguesas de Paralisia Cerebral, editor. 2018. 87

14. Day S, Reynolds R, Kush S. Extrapolating published survival curves to obtain evidence-based estimates of life expectancy in cerebral palsy. Developmental Medicine \& Child Neurology. 2015;57(12):1105-18.

15. Haak P, Lenski M, Hidecker M-J, Li M, Paneth N. Cerebral palsy and aging. Developmental Medicine \& Child Neurology. 2009;51(4):16-23.

16. Figueroa M, Rojas C, Barja S. Morbimortality associated to nutritional status and feeding path in children with cerebral palsy. Revista Chilena De Pediatria. 2017;88(4):478-86.

17. Himmelmann K, Sundh V. Survival with cerebral palsy over five decades in western 
Sweden. Developmental Medicine \& Child Neurology. 2015;57:762-7.

18. Blair E, Langdon K, Mclntyre S, Lawrence D, Watson L. Survival and mortality in cerebral palsy: observations to the sixth decade from a data linkage study of a total population register and national death index. BMC Neurology. 2019;19(1):1-11. 19. Ryan J, Peterson M, Ryan N, Smith K, O'connell N, Liverani S, et al. Mortality due to cardiovascular disease, respiratory disease, and cancer in adults with cerebral palsy. Developmental Medicine \& Child Neurology. 2019;61(8):924-8.

20. Landes S. Mortality risk among adults with cerebral palsy: alarming findings and lingering questions. Developmental Medicine \& Child Neurology. 2019;61(8):860-1.

21. Herrera-Anaya E, Angarita-Fonseca A, Herrera-Galindo VM, Martínez-Marín R, Rodríguez-Bayona C. Association between gross motor function and nutritional status in children with cerebral palsy: a cross-sectional study from Colombia. Developmental Medicine and Child Neurology. 2016;58(9):936-41.

22. Gulati S, Sondhi V. Cerebral palsy: an overview. Indian Journal of Pediatrics. 2018;85(11):1006-16.

23. Wort U, Nordmark E, Wagner P, Düppe H, Westbom L. Fractures in children with cerebral palsy: a total population study. Developmental Medicine and Child Neurology. 2013;55(9):821-6.

24. Pinto W, Alves LAC, Mendes FM, Ciamponi AL. The nutritional state of children and adolescents with cerebral palsy is associated with oral motor dysfunction and social conditions: a cross sectional study. BMC Neurology. 2016;16(1):1-7.

25. Kakooza-Mwesige A, Forssberg H, Eliasson A-C, Tumwine JK. Cerebral palsy in children in Kampala, Uganda: clinical subtypes, motor function and co-morbidities. BMC Research Notes. 2015;8(166):1-10.

26. Caramico-Favero D, Guedes Z, Morais M. Food intake, nutritional status and gastrointestinal symptoms in children with cerebral palsy. Arquivos De Gastroenterologia. 2018;55(4):352-7.

27. Stanek J, Emerson J, Murdock F, Petroski G. Growth characteristics in cerebral palsy subtypes: a comparative assessment. Developmental Medicine And Child Neurology. 2016;58(9):931-5.

28. Polack S, Adams M, O'banion D, Baltussen M, Asante S, Kerac M, et al. Children with cerebral palsy in Ghana: malnutrition, feeding challenges, and caregiver quality of life. Developmental Medicine \& Child Neurology. 2018;60(9):914-21.

29. Norte A, Alonso C, Martínez-Sanz JM, Gutierrez-Hervas A, Sospedra I. Nutritional status and cardiometabolic risk factors in institutionalized adults with cerebral palsy. Medicina. 2019;55(5):1-10.

30. Dahlseng M, Andersen G, Andrada M, Arnaud C, Balu R, de La Cruz J, et al. Gastrostomy tube feeding of children with cerebral palsy: Variation across six European countries. Developmental Medicine \& Child Neurology. 2012;54(10):938-44.

31. Mclntyre S, Badawi N, Goldsmith S, Hines M, Karlsson P, Mclntyre S, et al Australian Cerebral Palsy Register Report 2013 [Internet]. Report of the Australian Cerebral Palsy Register. Australia; 2013. Available from: https://www.cerebralpalsy. org.au/wp-content/uploads/2013/04/ACPR Report_Web_2013.pdf

32. Holden C, MacDonald A, Wharton B. Nutrition and child health. Nursing RC of, editor. Great Britain: Baillière Tindall; 2000. 307.

33. Finbråten A, Martins C, Andersen G, Skranes J, Brannsether B, Júlíusson P, et al. Assessment of body composition in children with cerebral palsy: a cross-sectional study in Norway. Developmental Medicine \& Child Neurology. 2015;57(9):858-64.

34. Kim H, Kang T, Park K, Kim J, Ahn H, Yim S. Which growth parameters can affect mortality in cerebral palsy? Plos One. 2019;14(6):1-15.

35. Oftedal S, Davies P, Boyd R, Stevenson R. Longitudinal growth, diet, and physical activity in young children with cerebral palsy. Pediatrics. 2016;138(4):1-8.

36. García-Iñiguez J, Vásquez-Garibay E, García-Contreras A, Romero-Velarde E, Troyo-Sanroman R. Assessment of anthropometric indicators in children with cerebral palsy according to the type of motor dysfunction and reference standard. Nutrición Hospitalaria. 2017;34(2):315-32269

37. Araújo L, Silva L. Anthropometric assessment of patients with cerebral palsy: which curves are more appropriate? Jornal de Pediatria. 2013;89(3):307-14.

38. Vega-Sanchez R, Gomez-Aguilar M, Haua K, Rozada G. Weight-based nutritional diagnosis of Mexican children and adolescents with neuromotor disabilities. BMC
Research Notes. 2012;5(218):1-7.

39. Benfer K, Weir K, Bell K, Ware R, Davies P, Boyd R. Food and fluid texture consumption in a population-based cohort of preschool children with cerebral palsy: relationship to dietary intake. Developmental Medicine and Child Neurology. 2015;57(11):1056-63.

40. Somerville H, Tzannes G, Wood J, Shun A, Hill C, Arrowsmith F, et al. Gastrointestinal and nutritional problems in severe developmental disability. Developmental Medicine and Child Neurology. 2008;50(9):712-6.

41. Motion S, Northstone K, Emond A, Stucke S, Golding J. Early feeding problems in children with cerebral palsy: weight and neuro-developmental outcomes. Developmental Medicine and Child Neurology. 2002;44(1):40-3.

42. Sullivan P, Lambert B, Rose M, Ford-Adams M, Johnson A, Griffiths P. Prevalence and severity of feeding and nutritional problems in children with neurological impairment: Oxford Feeding Study. Developmental Medicine and Child Neurology. 2000;42(10):674-80. 43. Vik T, Skrove M, Døllner H, Helland G. Spisevansker og vekstforstyrrelser blant barn med cerebral parese i Sør- og Nord-Trøndelag. Tidsskr Nor Lægeforen. 2001;13(121):1570-4. 44. Sangermano M, D’Aniello R, Massa G, Albano R, Pisano P, Budetta M, et al. Nutritional problems in children with neuromotor disabilities: an Italian case series. Italian Journal of Pediatrics. 2014;40(61):1-5.

45. Bell K, Benfer K, Ware R, Patrao T, Garvey J, Arvedson J, et al. Development and validation of a screening tool for feeding/swallowing difficulties and undernutrition in children with cerebral palsy. Developmental Medicine \& Child Neurology. 2019;61(10):1175-81. 46. Craig G, Carr L, Cass H, Hastings R, Lawson M, Reilly S, et al. Health, surgical, and health outcomes of gastrostomy feeding. Developmental Medicine \& Child Neurology. 2006;48:353-60.

47. Benfer K, Weir K, Ware R, Davies P, Arvedson J, Boyd R, et al. Parent-reported indicators for detecting feeding and swallowing difficulties and undernutrition in preschool-aged children with cerebral palsy. Developmental Medicine \& Child Neurology. 2017;59(11):1181-7.

48. Benfer K, Weir K, Bell K, Ware R, Davies P, Boyd R. The Eating and Drinking Ability Classification System in a population-based sample of preschool children with cerebral palsy. Developmental Medicine \& Child Neurology. 2017;59(6):647-54.

49. Gomes V, Campos M, Gregório M. Relação entre função motora, competências alimentares e peso de crianças e adolescentes com paralisia cerebral. Acta Portuguesa de Nutrição. 2016;6:20-3.

50. Sullivan P, Juszczak E, Lambert B, Rose M, Ford-Adams M, Johnson A. Impact of feeding problems on nutritional intake and growth: Oxford Feeding Study II. Developmental Medicine and Child Neurology. 2002;44(7):461-7.

51. Silvério CC, Henrique CS. Indicadores da evolução do paciente com paralisia cerebral e disfagia orofaríngea após intervenção terapêutica. Revista Da Sociedade Brasileira De Fonoaudiologia. 2009;14(3):381-6.

52. Campos M, Kent-Smith L, Santos C. Efeito das competências alimentares no estado nutricional de crianças portadoras de paralisia cerebral. Acta Médica Portuguesa. 2007;20(1):21-7.

53. Troughton K, Hill A. Relation between objectively measured feeding competence and nutrition in children with cerebral palsy. Developmental Medicine and Child Neurology. 2001;43(3):187-90.

54. Hung JW, Hsu TJ, Wu PC, Leong CP. Risk factors of undernutrition in children with spastic cerebral palsy. Chang Gung Medical Journal. 2003;26(6):425-32.

55. Caselli T, Lomazi E, Montenegro M, Bellomo-Brandão M. Assessment of nutritional status of children and adolescents with spastic quadriplegic cerebral palsy. Arquivos de Gastroenterologia. 2017;54(3):201-5

56. Speyer R, Cordier R, Kim JH, Cocks N, Michou E, Wilkes-Gillan S. Prevalence of drooling, swallowing, and feeding problems in cerebral palsy across the lifespan: a systematic review and meta-analyses. Dev Med Child Neurol. 2019;61(11):1249-58. 57. Menezes E da C, Santos FAH, Alves FL. Cerebral palsy dysphagia: a systematic review. Rev CEFAC. 2017;19(4):565-74.

58. Novak I, Morgan C, Fahey M, Finch-Edmondson M, Galea C, Hines A, et al. State of the Evidence Traffic Lights 2019: Systematic Review of Interventions for Preventing and Treating Children with Cerebral Palsy. Curr Neurol Neurosci Rep. 2020;20(2). 